\title{
Basic physical examination: a valid expectation, not an option
}

\author{
RJ MacFadyen \\ Consultant Cardiologist, Departments of Medicine and Cardiology, and University of Birmingham Centre for Cardiovascular Sciences, \\ Birmingham, UK
}

KEYWORDS TOS Study, physical examination, patient recollection, effective diagnostic triage

DECLARATION OF INTERESTS RJ MacFadyen is the Clinical Editor of the Journal of the Royal College of Physicians of Edinburgh. Although working in one of the institutions cited in this report, he had no awareness of this study nor involvement in its design, completion or results. The author has served as a specialist advisor to GMC fitness to practice panels. He is also a PACES Examiner.

\author{
Correspondence to RJ MacFadyen \\ City Hospital, Dudley Road, \\ Birmingham BI8 7QH, UK
}

tel. +44 (0)I2I $5074476 / 5634$

e-mail robert.macfadyen@nhs.net
In this issue, we include a pilot study which examined the completeness of physical examination of inpatients referred for specialist neurological assessment.' The findings, based upon patient recollection of diagnostic tools used during their initial medical assessment, suggest significant omissions in basic examination which have the potential to compromise the quality and safety of care delivered. In publishing this paper, we recognise it is based on a small scale study, with limitations, but believe these preliminary findings have wider implications for all in medicine, remind us of the importance of conducting physical examination ${ }^{2}$ and could be of value in directing future research in this area and defining the quality of individual medical practice.

The doctor-patient relationship involves a unique privilege awarded by patients to their doctor, that of permission for personal examination. Patients expect this and the assumption is that systematic examination facilitates diagnosis. This is a fundamental principle of medical teaching. There is evidence from the MRCP(UK) PACES exam ${ }^{3}$ that clinical examination skills among candidates are lacking and the most common reason for UK Trainees to fail PACES is the inability to pick up adequate marks for Skill B (identifying clinical signs).

Failure to complete a full examination may be defensible in urgent clinical emergency such as a need for immediate resuscitation, cardiopulmonary arrest or an environmental danger to the patient or doctor, however in any other setting it means missed opportunities to uncover latent disease or to target investigational studies appropriately. Even the easy availability of specialist advice or of powerful state of the art investigations should not exonerate busy doctors from performing their basic duty. Omitting basic examination is a fundamentally unacceptable standard of practice. This and other aspects of performance should fall within the remit of revalidation for any medical practitioner. ${ }^{4}$
Focusing on the Nicholl paper highlights increasing concerns that patients are not being given the benefit of a complete basic physical examination. While these observations clearly need expansion and corroboration in a variety of settings, they do raise questions and issues that should be addressed now, particularly when doctors and healthcare providers are under increasing scrutiny to ensure that the quality standards of care are maintained. It is also timely, given the work being carried out by MRCP(UK) on the relevance of clinical examination. Have we moved too far away from meeting basic responsibilities to the patient of a structured physical examination?

\section{IMPACT OF TARGETED EXAMINATION ON DIAGNOSTIC TRIAGE}

We already see the application of a range of sensitive but non-specific diagnostic tests used in community-wide screening to potentially identify a range of treatable diseases. ${ }^{5}$ Early detection without targeting is inefficient and less satisfactory for patients regardless of disease. Also, screening is not without harm and can be expensive for healthcare systems, such as the NHS. In contrast, the application of basic standards of symptomatic and examination triage might dramatically improve costeffectiveness, for patients and the population. Were structured physical examination used to triage such activity (and to avoid unnecessary laboratory tests) the cost savings could be significant and a critical adjunct to effectiveness. However, such tiered examinations need ongoing structured scientific assessment.

\section{KNOWLEDGE AND SKILLS}

There will always be a wide range of skill levels in examination, but routine completion of an evidencebased sequence is not complex; when structured, the 
findings facilitate the immediate management and the selection of appropriate investigations. Proficiency in recognising or excluding relevant clinical signs is surely the most credible and basic element of the required standard of medical practice - at qualification, in higher professional examinations and for continuous professional development. The importance of clinical assessment is emphasised at all levels in guideline documents, clinical commentaries, case reports and diagnostic series. But is this information being communicated at the right time, in the right place and to the right people to ensure patients are correctly managed?

\section{CONFIDENCE IN PERFORMING COMPLETE PHYSICAL EXAMINATIONS}

If doctors lack skills and knowledge, this will impact their level of competence in performing routine examination. What can we do to promote and underline competence in doctors?

\section{ASSESSMENT}

Huge resources are placed into the training and review of individual doctors yet practice standards are difficult to assess. Summative examinations incorporating structured assessments of skill in physical examination are a feature of higher medical training yet occur in isolation. New levels of review and monitoring will be required to track the activity of individual practitioners. For example, digital systems that monitor the number, duration and detail of individual medical contacts and decision-making do exist, offering exciting tools for real time quality assessment and a role in the validation of quality of care. ${ }^{6}$ From some of the issues raised above, it is clear that there are a number of areas for improvement that might be achieved by supportive regular review and continuing medical education.

Doctors are individually responsible at all stages in their professional career for their own activity and decisions. Current methods for recording learning and competencies must be supplemented by a drive to define quality guidelines for how individual doctors practice (trained and trainees) and a method for both establishing and monitoring them. Revalidation provides an excellent opportunity for reinforcing the standards for those who are not aware, convinced or who have perhaps lapsed into substandard routines. Perhaps the consequences of non-compliance need to be more stringent (i.e. linked to salaries or re-grading) to ensure that the goal of every doctor should be quality of care for patients, not an institutional activity or quota-filling. Deferring to others the responsibility for diagnosis, or inaction, is a dereliction of duty and should be actively engineered out of modern medical practice.

This is an opportunity for the UK to take the lead in commissioning research in this area, to ensure that all doctors get back to the basics of performing complete structured physical examinations of all patients at the front door. The Nicholl et al. study has shown us that our patients can clearly serve a role in this partnership in defining their own quality of medical care and that of their doctor.

\section{REFERENCES}

I Nicholl D, Yap CP, Cahill V et al The TOS study: can we use our patients to help improve clinical assessment? J $R$ Coll Physicians Edinb 2012; 42:306-10.

2 Verghese A, Horwitz RI. In praise of the physical examination. BM] 2009; 339:b5448. http://dx.doi.org// 0.1 I 36/bmj.b5448

3 Elder AT, McAlpine L, Bateman $\mathrm{N}$ et al. Changing PACES: developments to the examination in 2009. Clin Med 20 I I; I I:23 I-4.

4 Health Committee, UK Parliament. Revalidation of doctors. London: The Stationery Office; $201 \mathrm{I}$ [cited 2012 Nov 6]. Available from: www.publications.parliament.uk/pa/cm20I0II/cmselect/ cmhealth/557/55702.htm

5 MacFadyen RJ. Referral for diagnosis: effectiveness not activity or expediency is a priority. J $R$ Coll Physicians Edinb 2012; 42:5-7. http://dx.doi.org//0.4997/JRCPE.2012.102

6 Schenarts PJ, Schenarts KD. Educational impact of the electronic medical record. J Surg Educ 2012; 69: 105-12. http://dx.doi. org/I0.10I6/j.jsurg.20II.10.008 